\title{
Generalized Metric Spaces Do Not Have the Compatible Topology
}

\author{
Tomonari Suzuki ${ }^{1,2}$ \\ ${ }^{1}$ Department of Basic Sciences, Faculty of Engineering, Kyushu Institute of Technology, Tobata, Kitakyushu 804-8550, Japan \\ ${ }^{2}$ Department of Mathematics, Faculty of Science, King Abdulaziz University, Jeddah, Saudi Arabia
}

Correspondence should be addressed to Tomonari Suzuki; suzuki-t@mns.kyutech.ac.jp

Received 12 May 2014; Accepted 9 July 2014; Published 4 August 2014

Academic Editor: Wei-Shih Du

Copyright (C) 2014 Tomonari Suzuki. This is an open access article distributed under the Creative Commons Attribution License, which permits unrestricted use, distribution, and reproduction in any medium, provided the original work is properly cited.

We study generalized metric spaces, which were introduced by Branciari (2000). In particular, generalized metric spaces do not necessarily have the compatible topology. Also we prove a generalization of the Banach contraction principle in complete generalized metric spaces.

\section{Introduction}

In 2000, Branciari in [1] introduced a very interesting concept whose name is " $\nu$-generalized metric space."

Definition 1 (see Branciari [1]). Let $X$ be a set, let $d$ be a function from $X \times X$ into $[0, \infty)$, and let $v \in \mathbb{N}$. Then $(X, d)$ is said to be a $\nu$-generalized metric space if the following hold:

(N1) $d(x, y)=0$ if and only if $x=y$ for any $x, y \in X$;

(N2) $d(x, y)=d(y, x)$ for any $x, y \in X$;

(N3) $d(x, y) \leq d\left(x, u_{1}\right)+d\left(u_{1}, u_{2}\right)+\cdots+d\left(u_{v}, y\right)$ for any $x, u_{1}, u_{2}, \ldots, u_{v}, y \in X$ such that $x, u_{1}, u_{2}, \ldots, u_{v}, y$ are all different.

Example 2. Every metric space $(X, d)$ is a 1-generalized metric space.

A 2-generalized metric space is also said to be a generalized metric space.

Definition 3 (see Branciari [1]). Let $X$ be a set and let $d$ be a function from $X \times X$ into $[0, \infty)$. Then $(X, d)$ is said to be ageneralized metric space if the following hold:

(G1) $d(x, y)=0$ if and only if $x=y$ for any $x, y \in X$.

(G2) $d(x, y)=d(y, x)$ for any $x, y \in X$.
(G3) $d(x, y) \leq d(x, u)+d(u, v)+d(v, y)$ for any $x, u, v, y \in$ $X$ such that $x, u, v, y$ are all different.

The concept of "generalized metric space" is very similar to that of "metric space." However, it is very difficult to treat this concept because $X$ does not necessarily have the topology which is compatible with $d$; see Example 7. So this concept is very interesting to researchers. See also $[2,3]$.

Motivated by the above, in this paper, we study generalized metric spaces. In particular, generalized metric spaces do not necessarily have the compatible topology. Also we prove a generalization of the Banach contraction principle in complete generalized metric spaces.

\section{2. $v$-Generalized Metric Space}

Throughout this paper we denote by $\mathbb{N}$ the set of all positive integers.

In this section, we study $\nu$-generalized metric space. In particular, we give examples in order to understand this concept deeply.

Lemma 4. Let $(X, \rho)$ be a bounded metric space and let $M$ be a real number satisfying

$$
\sup \{\rho(x, y): x, y \in X\} \leq M \text {. }
$$


Let $A$ and $B$ be two subsets of $X$ with $X=A \cup B$ and $A \cap B=\varnothing$. Define a function d from $X \times X$ into $[0, \infty)$ by

$$
\begin{array}{ll}
d(x, x)=0 & \\
d(x, y)=d(y, x)=\rho(x, y) & \text { if } x \in A, y \in B \\
d(x, y)=M & \text { otherwise. }
\end{array}
$$

Then $(X, d)$ is a generalized metric space.

Proof. (N1) and (N2) are obvious. Let us prove (N3). Let $x, y, u, v \in X$ be all different. Put

$$
t=d(x, u)+d(u, v)+d(v, y) .
$$

In the case where $t \geq M$, (N3) holds because $d(x, y) \leq M$. In the other case, where $t<M$, without loss of generality, we may assume $x \in A$. Then we have $v \in A$ and $u, y \in B$ from the definition of $d$. Hence,

$$
\begin{aligned}
d(x, y) & =\rho(x, y) \leq \rho(x, u)+\rho(u, v)+\rho(v, y) \\
& =d(x, u)+d(u, v)+d(v, y) .
\end{aligned}
$$

Thus (N3) holds.

Definition 5. Let $(X, d)$ be a $\nu$-generalized metric space. Then a net $\left\{x_{\alpha}\right\}$ is said to converge to $x$ if and only if $\lim _{\alpha} d\left(x, x_{\alpha}\right)=$ 0 .

Definition 6. Let $X$ be a topological space with topology $\tau$. Let $d$ be a function from $X \times X$ into $[0, \infty)$ satisfying (N1)-(N3) with some $v \in \mathbb{N}$. Then $\tau$ is compatible with $d$ if and only if the following are equivalent for any net $\left\{x_{\alpha}\right\}$ in $X$ and $x \in X$ :

(a) $\lim _{\alpha} d\left(x, x_{\alpha}\right)=0$.

(b) $\left\{x_{\alpha}\right\}$ converges to $x$ in $\tau$.

The following is a very important example.

Example 7. Let

$$
X=\{(0,0)\} \cup((0,1] \times[0,1]) .
$$

Define a function $d$ from $X \times X$ into $[0, \infty)$ by

$$
\begin{array}{ll}
d(x, x)=0 & \\
d((0,0),(s, 0))=d((s, 0),(0,0))=s, & \text { if } s \in(0,1] \\
d((s, 0),(p, q)) & \\
\quad=d((p, q),(s, 0))=|s-p|+q, & \text { if } s, p, q \in(0,1] \\
d(x, y)=3, & \text { otherwise. }
\end{array}
$$

Then the following hold:

(i) $(X, d)$ is not a metric space;

(ii) $(X, d)$ is a generalized metric space; (iii) $X$ does not have a topology which is compatible with $d$.

Proof. Since

$$
\begin{aligned}
& d((0,0),(1,0))+d((1,0),(1,1)) \\
& \quad=1+1=2<3=d((0,0),(1,1)),
\end{aligned}
$$

$(X, d)$ is not a metric space. Define a metric $\rho$ on $X$ by

$$
\rho((s, t),(p, q))=|s-p|+|t-q|,
$$

for $(s, t),(p, q) \in X$. Put

$$
A=\{(0,0)\} \cup((0,1] \times(0,1]), \quad B=(0,1] \times\{0\} .
$$

Then $d$ is equal to the $d$ defined by Lemma 4 with $M=3$. Therefore, $(X, d)$ is a generalized metric space. In order to show (iii), we will show that the following does not hold.

If a net $\left\{x_{\alpha}\right\}_{\alpha \in D}$ converges to $x$ and for every $\alpha \in D$ a net $\left\{x_{(\alpha, \beta)}\right\}_{\beta \in E_{\alpha}}$ converges to $x_{\alpha}$, then $\left\{x_{(\alpha, \gamma)}\right\}_{(\alpha, \gamma) \in D \times \prod\left\{E_{\alpha}: \alpha \in D\right\}}$ has a subnet converging to $x$; see [4, page 77].

We have that $\{(1 / \ell, 0)\}_{\ell}$ converges to $(0,0)$ and $\{(1 / \ell, 1 / m)\}_{m}$ converges to $(1 / \ell, 0)$ for every $\ell \in \mathbb{N}$. However, since $d((0,0),(1 / \ell, 1 / m))=3$ for $(\ell, m) \in \mathbb{N}^{2}$, a net $\{(1 / \ell, 1 / \gamma(\ell))\}_{(\ell, \gamma)}$ does not converge to $(0,0)$. Therefore there does not exist a topology which is compatible with $d$.

Remark 8. For $(\alpha, \gamma) \in D \times \prod\left\{E_{\alpha}: \alpha \in D\right\}, x_{(\alpha, \gamma)}=x_{(\alpha, \gamma(\alpha))}$. For $\left(\alpha_{1}, \gamma_{1}\right),\left(\alpha_{2}, \gamma_{2}\right) \in D \times \prod\left\{E_{\alpha}: \alpha \in D\right\},\left(\alpha_{1}, \gamma_{1}\right) \leq\left(\alpha_{2}, \gamma_{2}\right)$ if and only if $\alpha_{1} \leq \alpha_{2}$ and $\gamma_{1}(\alpha) \leq \gamma_{2}(\alpha)$ for any $\alpha \in D$.

Remark 9. Indeed, let $\tau$ be the topology induced by a subbase:

$$
\{S(x, r): x \in X, r>0\},
$$

where $S(x, r)=\{y \in X: d(x, y)<r\}$. Since

$$
\begin{aligned}
S & ((0,0), 2) \cap S((1,0), 2) \\
& =([0,1] \times\{0\}) \cap(\{(0,0),(1,0)\} \cup((0,1] \times(0,1])) \\
& =\{(0,0),(1,0)\},
\end{aligned}
$$

we have

$$
S((0,0), 2) \cap S((1,0), 1)=\{(1,0)\} .
$$

Hence $\{(1,0)\}$ is an open neighborhood of $(1,0)$. So a sequence $\{(1,1 / n)\}$ does not converge to $(1,0)$ in $\tau$. Since $\lim _{n} d((1,0),(1,1 / n))=0, \tau$ is not compatible with $d$.

We can easily make an example of a $\nu$-generalized metric space which is not a $\mu$-generalized metric space for $\mu<\nu$.

Example 10. Put $X=\mathbb{N}$ and let $v \in \mathbb{N}$ satisfy $v \geq 2$. Define a function $d$ from $X \times X$ into $[0, \infty)$ by

$$
\begin{array}{ll}
d(x, x)=0, & \\
d(1, s)=d(s, 1)=v+1, & \text { if } s \in \mathbb{N} \backslash\{1,2\}, \\
d(x, y)=1, & \text { otherwise. }
\end{array}
$$

Then the following hold: 
(i) $(X, d)$ is not a $\mu$-generalized metric space for $\mu \in \mathbb{N}$ with $\mu<\nu$;

(ii) $(X, d)$ is a $\mu$-generalized metric space for $\mu \in \mathbb{N}$ with $\mu \geq v$.

Proof. (N1) and (N2) obviously hold. Let $\mu \in \mathbb{N}$ satisfy $\mu<\nu$. Since

$$
\sum_{j=1}^{\mu+1} d(j, j+1)=\mu+1<\nu+1=d(1, \mu+2),
$$

(N3) does not hold. So $(X, d)$ is not a $\mu$-generalized metric space. Let $\mu \in \mathbb{N}$ satisfy $\mu \geq \nu$. Let $x, u_{1}, u_{2}, \ldots u_{\mu}, y \in X$ be all different. Then we have

$$
\begin{aligned}
d(x, y) \leq & \nu+1 \leq \mu+1 \leq d\left(x, u_{1}\right) \\
& +d\left(u_{1}, u_{2}\right)+\cdots+d\left(u_{\mu}, y\right) .
\end{aligned}
$$

Thus (N3) holds. Hence $(X, d)$ is a $\mu$-generalized metric space.

We give some definitions. The reason of these definitions is that $(X, d)$ does not necessarily have the topology which is compatible with $d$. So $(X, d)$ does not necessarily have the uniformity which is compatible with $d$.

Definition 11. Let $(X, d)$ be a $\nu$-generalized metric space.

(a) A sequence $\left\{x_{j}\right\}$ is said to be Cauchy if and only if $\lim _{j} \sup _{m>j} d\left(x_{j}, x_{m}\right)=0$.

(b) $X$ is said to be complete if and only if every Cauchy sequence converges to some point in $X$.

(c) $X$ is said to be Hausdorff if and only if $\lim _{j} d\left(x, x_{j}\right)=$ $\lim _{j} d\left(y, x_{j}\right)=0$ implies $x=y$.

Lemma 12. Let $(X, d)$ be a v-generalized metric space and let $x, u_{1}, \ldots, u_{v}, y \in X$ such that $x, u_{1}, \ldots, u_{v}$ are all different and $u_{1}, \ldots, u_{v}, y$ are all different. Then

$$
d(x, y) \leq d\left(x, u_{1}\right)+d\left(u_{1}, u_{2}\right)+\cdots+d\left(u_{v}, y\right)
$$

holds.

Proof. In the case where $x=y$, the conclusion obviously holds from (N1). In the other case, where $x \neq y$, the conclusion obviously holds from (N3).

\section{The CJM Fixed Point Theorem}

In this section, we generalize the CJM fixed point theorem; see Ćirić [5], Jachymski [6], and Matkowski [7, 8].

Theorem 13. Let $(X, d)$ be a complete $\nu$-generalized metric space and let $T$ be a CJM contraction on $X$; that is, the following hold:

(i) for every $\varepsilon>0$, there exists $\delta>0$ such that $d(x, y)<$ $\varepsilon+\delta$ implies $d(T x, T y) \leq \varepsilon$ for any $x, y \in X ;$

(ii) $x \neq y$ implies $d(T x, T y)<d(x, y)$ for any $x, y \in X$.
Then $T$ has a unique fixed point $z$ of $T$. Moreover, $\lim _{j} d\left(T^{j} x, z\right)=0$ for any $x \in X$.

Proof. We first note that $T$ is nonexpansive by (ii); that is

$$
d(T x, T y) \leq d(x, y)
$$

for any $x, y \in X$. Fix $u \in X$ and define a sequence $\left\{u_{j}\right\}$ in $X$ by $u_{j}=T^{j} u$ for $j \in \mathbb{N}$. We next show that $\left\{u_{j}\right\}$ converges to a fixed point of $T$, dividing the following three cases:

(a) there exists $n \in \mathbb{N}$ such that $u_{n+1}=u_{n}$;

(b) $u_{j+1} \neq u_{j}$ for all $j \in \mathbb{N}$ and there exist $m, n \in \mathbb{N}$ such that $m+2 \leq n$ and $u_{m}=u_{n}$;

(c) $u_{1}, u_{2}, \ldots$ are all different.

In the first case, $u_{n}$ is a fixed point of $T$. By (N1), $\left\{u_{j}\right\}$ converges to $u_{n}$. In the second case, from (ii), we have $\left\{d\left(u_{j}, u_{j+1}\right)\right\}$ is strictly decreasing. So, since $u_{m+1}=u_{n+1}$, we have

$$
d\left(u_{m}, u_{m+1}\right)=d\left(u_{n}, u_{n+1}\right)<d\left(u_{m}, u_{m+1}\right) .
$$

This is a contradiction. Thus, the second case cannot be possible. In the third case, from (ii), we have $\left\{d\left(u_{j}, u_{j+k}\right)\right\}$ is strictly decreasing for any $k \in \mathbb{N}$. So $\left\{d\left(u_{j}, u_{j+k}\right)\right\}$ converges to some $\varepsilon_{1} \geq 0$. Then we note that $d\left(u_{j}, u_{j+k}\right)>\varepsilon_{1}$ for every $j \in \mathbb{N}$. Arguing by contradiction, we assume $\varepsilon_{1}>0$. From (i), there exists $\delta_{1}>0$ such that

$$
d(x, y)<\varepsilon_{1}+\delta_{1} \quad \text { implies } d(T x, T y) \leq \varepsilon_{1} .
$$

From the definition of $\varepsilon_{1}$, there exists $n \in \mathbb{N}$ such that $d\left(u_{n}, u_{n+k}\right)<\varepsilon_{1}+\delta_{1}$. Then we have $d\left(u_{n+1}, u_{n+k+1}\right) \leq \varepsilon_{1}$. This is a contradiction. Therefore we obtain $\varepsilon_{1}=0$. That is, $\lim _{j} d\left(u_{j}, u_{j+k}\right)=0$ holds for any $k \in \mathbb{N}$. Thus

$$
\lim _{j \rightarrow \infty} \max \left\{d\left(u_{j}, u_{j+k}\right): k=1,2, \ldots, v+1\right\}=0
$$

holds. Fix $\varepsilon_{2}>0$. Then, by (i), there exists $\delta_{2} \in\left(0, \varepsilon_{2}\right)$ such that

$$
d(x, y)<\varepsilon_{2}+2 \nu \delta_{2} \quad \text { implies } d(T x, T y) \leq \varepsilon_{2} .
$$

Let $\ell \in \mathbb{N}$ such that

$$
\max \left\{d\left(u_{j}, u_{j+k}\right): k=1,2, \ldots, v+1\right\}<\delta_{2},
$$

for all $j \in \mathbb{N}$ with $j \geq \ell$. We will show

$$
d\left(u_{\ell}, u_{\ell+m}\right)<\varepsilon_{2}+v \delta_{2},
$$

for $m \in \mathbb{N}$ by induction. For $m=1,2, \ldots, v+1$, we have

$$
d\left(u_{\ell}, u_{\ell+m}\right)<\delta_{2}<\varepsilon_{2}+\nu \delta_{2}
$$

and, thus, (23) holds. We assume (23) holds for some $m \in \mathbb{N}$ with $m>v$. We have, by (N3),

$$
\begin{aligned}
& d\left(u_{\ell+v}, u_{\ell+m}\right) \\
& \quad \leq \sum_{j=1}^{v} d\left(u_{\ell+j}, u_{\ell+j-1}\right)+d\left(u_{\ell}, u_{\ell+m}\right) \\
& \quad<\nu \delta_{2}+\varepsilon_{2}+\nu \delta_{2}=\varepsilon_{2}+2 \nu \delta_{2} .
\end{aligned}
$$


Hence $d\left(u_{\ell+v+1}, u_{\ell+m+1}\right) \leq \varepsilon_{2}$. We put

$$
\alpha= \begin{cases}d\left(u_{\ell}, u_{\ell+v+1}\right) & \text { if } v=1 \\ d\left(u_{\ell}, u_{\ell+1}\right)+d\left(u_{\ell+1}, u_{\ell+v+1}\right) & \text { if } v=2 \\ \sum_{j=\ell}^{\ell+v-2} d\left(u_{j}, u_{j+1}\right)+d\left(u_{\ell+v-1}, u_{\ell+\nu+1}\right) & \text { if } v>2 .\end{cases}
$$

We note $\alpha<\nu \delta_{2}$. By (N3), we have

$$
d\left(u_{\ell}, u_{\ell+m+1}\right) \leq \alpha+d\left(u_{\ell+v+1}, u_{\ell+m+1}\right)<\nu \delta_{2}+\varepsilon_{2} .
$$

Thus, (23) holds for $m:=m+1$. So, by induction, (23) holds for every $m \in \mathbb{N}$. Therefore we have shown

$$
\lim _{\ell \rightarrow \infty} \sup _{\ell<m}\left(u_{\ell}, u_{m}\right) \leq \varepsilon_{2}+\nu \delta_{2}<(\nu+1) \varepsilon_{2} .
$$

Since $\varepsilon_{2}>0$ is arbitrary, we obtain that $\left\{u_{j}\right\}$ is Cauchy. Since $X$ is complete, $\left\{u_{j}\right\}$ converges to some point $z \in X$. We have by Lemma 12 and the nonexpansiveness of $T$

$$
\begin{aligned}
& d(z, T z) \\
& \quad \leq\left(d\left(z, u_{m+1}\right)+\sum_{j=1}^{\nu-1} d\left(u_{m+j}, u_{m+j+1}\right)+d\left(u_{m+v}, T z\right)\right) \\
& \quad \leq\left(d\left(z, u_{m+1}\right)+\sum_{j=1}^{\nu-1} d\left(u_{m+j}, u_{m+j+1}\right)+d\left(u_{m+\nu-1}, z\right)\right),
\end{aligned}
$$

for sufficiently large $m \in \mathbb{N}$. As $m$ tends to $\infty$, we obtain $d(z, T z)=0$. Thus, $z$ is a fixed point of $T$. The uniqueness of the fixed point is obviously followed by (ii).

Remark 14. In [9], there is another fixed point theorem which is independent of Theorem 13.

By Theorem 13, we obtain a generalization of the Banach contraction principle $[10,11]$.

Corollary 15 (see Branciari [1]). Let $(X, d)$ be a complete $v$ generalized metric space and let $T$ be a contraction on $X$; that is, there exists $r \in[0,1)$ such that

$$
d(T x, T y) \leq r d(x, y),
$$

for any $x, y \in X$. Then $T$ has a unique fixed point $z$ of $T$. Moreover, $\lim _{j} d\left(T^{j} x, z\right)=0$ for any $x \in X$.

Remark 16. The authors in [12] stated the proof in [1] is incorrect and gave a proof under the assumption that $(X, d)$ is Hausdorff and $v=2$. See also [13].

In order to show that Theorem 13 is a generalization of Theorem 3.1 in [14], we prove the following. See also [15]. The idea on the proof of the following proposition appears in $[16$, 17].

Proposition 17. Let $(X, d)$ be a v-generalized metric space and let $T$ be a mapping on $X$. Assume that there exist functions $\varphi, \psi$ from $[0, \infty)$ into $[0, \infty)$ such that the following hold: (i) $\psi(d(T x, T y)) \leq \psi(d(x, y))-\varphi(d(x, y))$ for any $x, y \in$ $X$;

(ii) $\psi$ is nondecreasing;

(iii) $\inf \varphi([s, t])>0$ for any $s, t \in(0, \infty)$ with $s<t$.

Then $T$ is a CJM contraction.

Proof. Since $\varphi(t)>0$ for any $t \in(0, \infty)$, (ii) of the definition of CJM contraction obviously holds. We will show (i) of the definition of CJM contraction. Fix $\varepsilon>0$. From (iii), we can put

$$
\eta:=\inf \{\varphi(t): \varepsilon \leq t \leq \varepsilon+1\}>0 .
$$

We choose $\delta \in(0,1)$ such that

$$
\psi(\varepsilon+\delta)<\lim _{t \rightarrow \varepsilon+0} \psi(t)+\eta .
$$

Let $x, y \in X$ satisfy $d(x, y)<\varepsilon+\delta$. In the case where $d(x, y)=$ 0 , we have $d(T x, T y)=0$ because $x=y$. In the case where $0<d(x, y) \leq \varepsilon$, we have

$$
\begin{aligned}
& \psi(d(T x, T y)) \\
& \quad \leq \psi(d(x, y))-\varphi(d(x, y))<\psi(d(x, y)) \leq \psi(\varepsilon),
\end{aligned}
$$

which implies $d(T x, T y)<\varepsilon$. In the other case, where $d(x, y)>\varepsilon$, we have

$$
\begin{aligned}
\psi( & (T(T x, T y)) \\
& \leq \psi(d(x, y))-\varphi(d(x, y)) \leq \psi(\varepsilon+\delta)-\eta \\
& <\lim _{t \rightarrow \varepsilon+0} \psi(t)+\eta-\eta=\lim _{t \rightarrow \varepsilon+0} \psi(t),
\end{aligned}
$$

which implies $d(T x, T y) \leq \varepsilon$. Hence we have $d(T x, T y) \leq \varepsilon$ in all cases. Therefore $T$ is a CJM contraction.

\section{Conflict of Interests}

The author declares that there is no conflict of interests regarding the publication of this paper.

\section{Acknowledgments}

The author wishes to express his sincere thanks to the referees for their careful reading and many suggestions. The author is supported in part by Grant-in-Aid for Scientific Research from Japan Society for the Promotion of Science.

\section{References}

[1] A. Branciari, "A fixed point theorem of Banach-Caccioppoli type on a class of generalized metric spaces," Publicationes Mathematicae Debrecen, vol. 57, no. 1-2, pp. 31-37, 2000.

[2] K. Włodarczyk and R. Plebaniak, "Leader type contractions, periodic and fixed points and new completivity in quasi-gauge spaces with generalized quasi-pseudodistances," Topology and its Applications, vol. 159, no. 16, pp. 3504-3512, 2012. 
[3] K. Włodarczyk and R. Plebaniak, "Contractions of Banach, Tarafdar, Meir-Keeler, Ćirić-Jachymski-Matkowski and Suzuki types and fixed points in uniform spaces with generalized pseudodistances," Journal of Mathematical Analysis and Applications, vol. 404, no. 2, pp. 338-350, 2013.

[4] S. Willard, General Topology, Dover Publications, New York, NY, USA, 2004.

[5] L. Ćirić, "A new fixed-point theorem for contractive mappings," Publications de l'Institut Mathématique, vol. 30, pp. 25-27, 1981.

[6] J. Jachymski, "Equivalent conditions and the Meir-Keeler type theorems," Journal of Mathematical Analysis and Applications, vol. 194, no. 1, pp. 293-303, 1995.

[7] M. Kuczma, B. Choczewski, and R. Ger, Iterative Functional Equations, vol. 32 of Encyclopedia of Mathematics and its Applications, Cambridge University Press, Cambridge, UK, 1990.

[8] J. Matkowski, "Fixed point theorems for contractive mappings in metric spaces," Časopis Pro Pěstování Matematiky, vol. 105, no. 4, pp. 341-344, 1980.

[9] A. Fora, A. Bellour, and A. Al-Bsoul, "Some results in fixed point theory concerning generalized metric spaces," Matematichki Vesnik, vol. 61, no. 3, pp. 203-208, 2009.

[10] S. Banach, "Sur les opérations dans les ensembles abstraits et leur application aux équations intégrales," Fundamenta Mathematicae, vol. 3, pp. 133-181, 1922.

[11] R. Caccioppoli, "Un teorema generale sullesistenza di elementi uniti in una transformazione funzionale," Rendiconti dell'Accademia Nazionale dei Lincei, vol. 11, pp. 794-799, 1930.

[12] I. R. Sarma, J. M. Rao, and S. S. Rao, "Contractions over generalized metric spaces," Journal of Nonlinear Science and its Applications, vol. 2, no. 3, pp. 180-182, 2009.

[13] B. Samet, "Discussion on "A fixed point theorem of BanachCaccioppoli type on a class of generalized metric spaces" by A. Branciari," Publicationes Mathematicae Debrecen, vol. 76, no. 34, pp. 493-494, 2010.

[14] H. Lakzian and B. Samet, "Fixed points for $(\psi, \phi)$-weakly contractive mappings in generalized metric spaces," Applied Mathematics Letters, vol. 25, no. 5, pp. 902-906, 2012.

[15] P. N. Dutta and B. S. Choudhury, "A generalisation of contraction principle in metric spaces," Fixed Point Theory and Applications, vol. 2008, Article ID 406368, 2008.

[16] T. Suzuki, "Meir-KEEler contractions of integral type are still Meir-KEEler contractions," International Journal of Mathematics and Mathematical Sciences, vol. 2007, Article ID 39281, 6 pages, 2007.

[17] T. Suzuki and C. Vetro, "Three existence theorems for weak contractions of Matkowski type," International Journal of Mathematics and Statistics, vol. 6, no. 10, pp. S110-S120, 2010. 


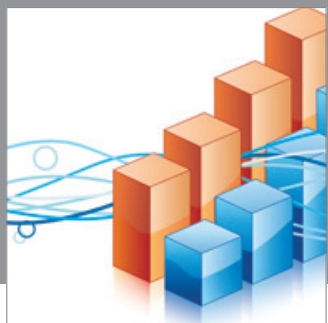

Advances in

Operations Research

mansans

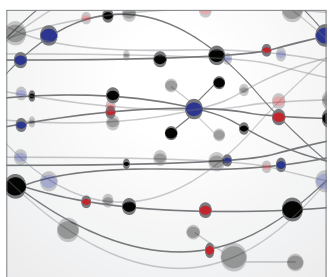

The Scientific World Journal
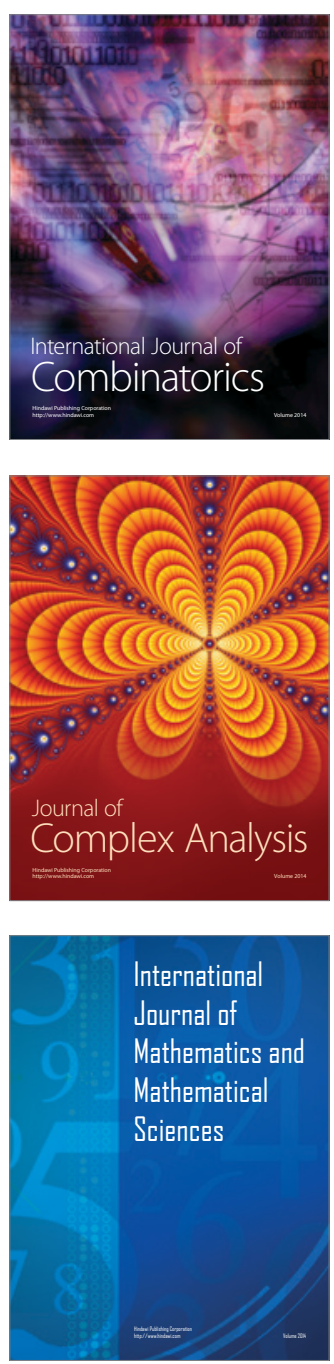
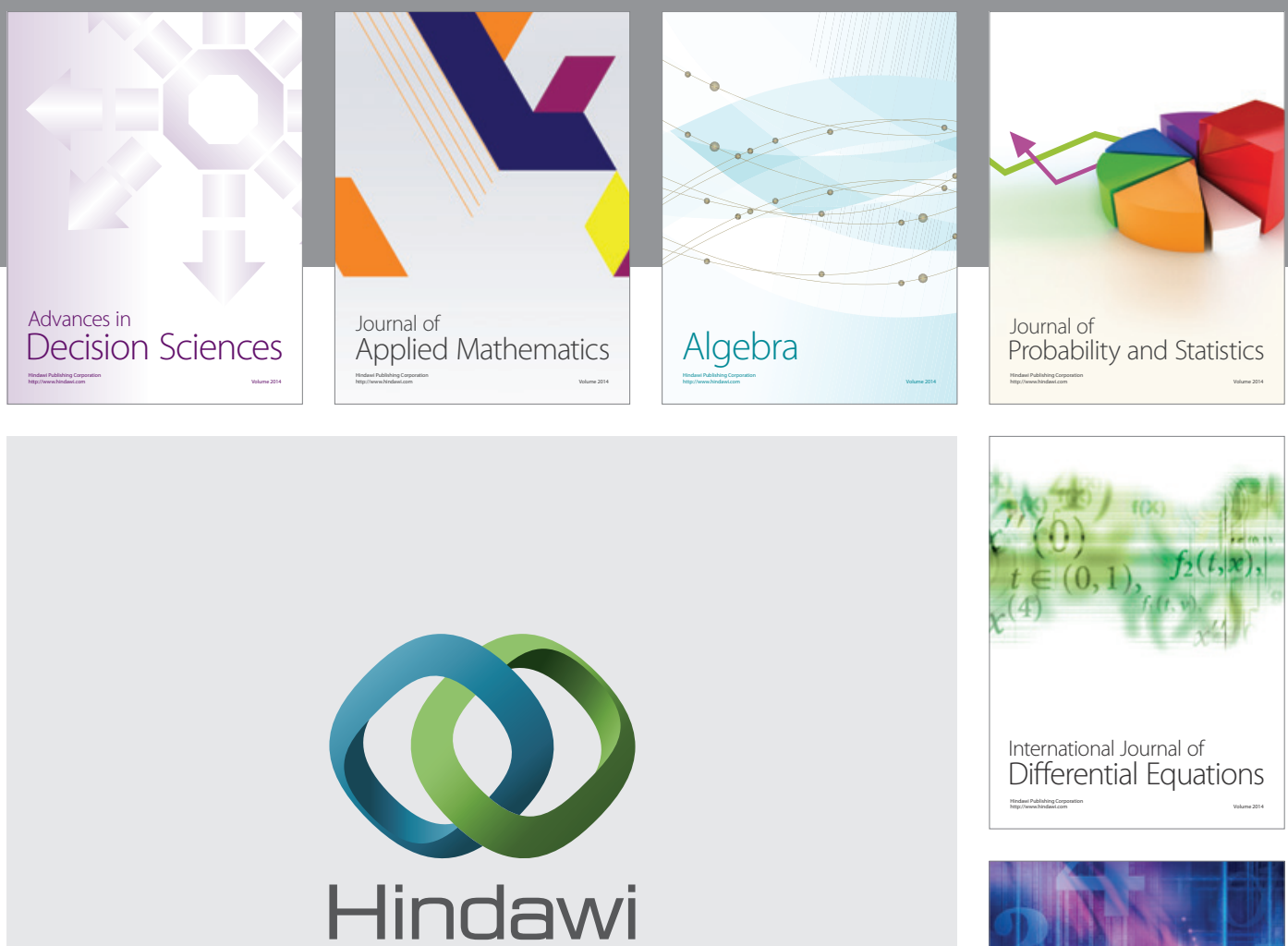

Submit your manuscripts at http://www.hindawi.com
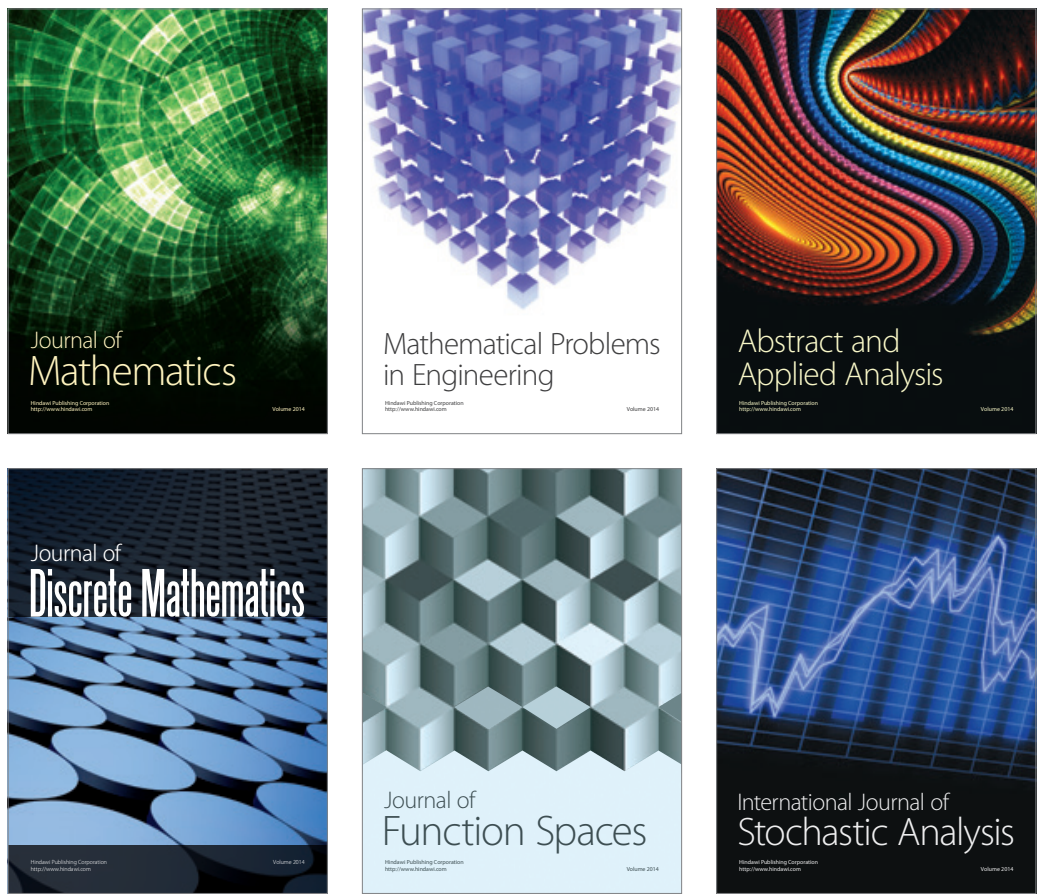

Journal of

Function Spaces

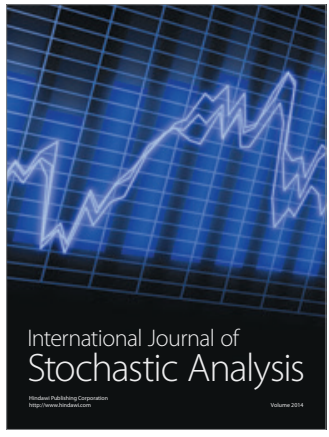

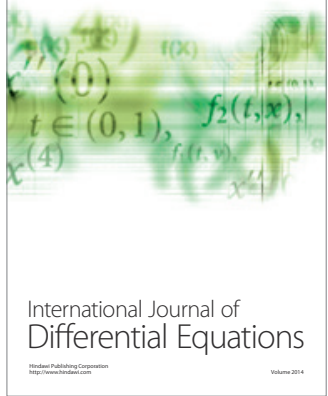
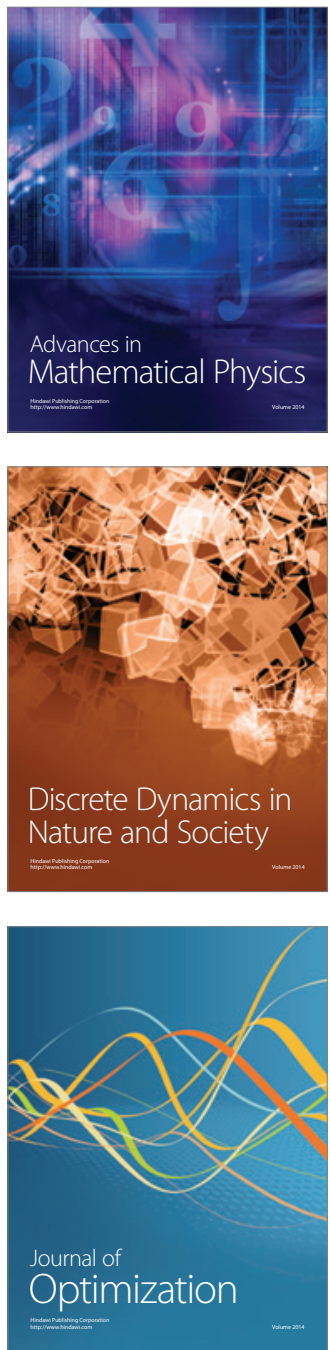